\title{
COVID-19 in decompensated cirrhosis
}

\author{
Mohamed Rela $^{1} \cdot$ Vaibhav Patil $^{1} \cdot$ Gomathy Narasimhan ${ }^{1} \cdot$ Dinesh Jothimani $^{1}$ (i)
}

Received: 4 June 2020 / Accepted: 6 September 2020 / Published online: 29 September 2020

(c) Asian Pacific Association for the Study of the Liver 2020

\section{Dear Editor}

COVID-19 is associated with higher mortality in patients pre-existing comorbidities. There has been several reports recently describing abnormalities in liver function tests (LFT) in COVID-19 patients ranging from very mild abnormalities in the non-cirrhotic patients to moderate elevation of liver enzymes in the cirrhotic patients $[1,2]$. Our recent review showed that $14-53 \%$ of patients with COVID-19 developed hepatic dysfunction and $2-11 \%$ of patients had underlying chronic liver disease [3].

In this context, we would like to report two of our patients with decompensated cirrhosis presented with acute-onchronic liver failure (ACLF) due to SARS-CoV-2 infection. Both patients had a rapidly progressive liver failure followed by respiratory failure and succumbed to the disease within days of the diagnosis.

Case 1 A 69-year-old gentleman with NASH cirrhosis and chronic portal vein thrombosis was diagnosed in March 2020 with a small hepatocellular carcinoma. His bilirubin was $8.8 \mathrm{mg} / \mathrm{dl}$, alanine amino transferase (ALT) $42 \mathrm{U} / \mathrm{L}$, albumin $2.6 \mathrm{~g} / \mathrm{dL}$, INR 1.54 and creatinine $1.28 \mathrm{mg} / \mathrm{dl}$. His Child Pugh score was 10 (Class C) and his model for end-stage liver disease (MELD) was 23. His HBsAg, anti$\mathrm{HBcAb}$, and anti-HCV antibodies were negative. He was being worked up for liver transplantation. He presented again in mid-April with 5 days history of abdominal distension, altered sensorium and breathing difficulty. His past medical history includes type 2 diabetes mellitus and hypertension. Clinical examination revealed jaundice, moderate ascites (grade 2), pedal edema and grade 2 encephalopathy. His respiratory rate was 28 per minute and oxygen saturation $92 \%$ on room air. His arterial blood gas showed $\mathrm{pH} 7 \cdot 314$, $\mathrm{pO}_{2} 55.4 \mathrm{~mm} \mathrm{Hg}$ and $\mathrm{pCO}_{2} 36 \cdot 8 \mathrm{mmHg}$. His $\mathrm{Hb} 12.1 \mathrm{~g} / \mathrm{dl}$,

Dinesh Jothimani

dinesh.jothimani@relainstitute.com

1 Institute of Liver Disease and Transplantation, Dr Rela Institute and Medical Centre, Bharath Institute for Higher Education and Research, Chennai, India white blood cell count 7780 cells $/ \mathrm{mm}^{3}$, differential count showed Neutrophils 6690 cells $/ \mathrm{mm}^{3}$ (86\%), lymphocytes $311 \mathrm{cells} / \mathrm{mm}^{3}(4.0 \%)$, and platelet count $139 \times 10^{9} / \mathrm{L}$. He was negative for Hepatitis A and Hepatitis E IgM antibodies. His serum bilirubin was $20.2 \mathrm{mg} / \mathrm{dl}$, ALT $58 \mathrm{U} / \mathrm{L}$, albumin $2.7 \mathrm{~g} / \mathrm{dL}$, International normalized ratio (INR) 2.82 and creatinine was $2.83 \mathrm{mg} / \mathrm{dl}$ (not on diuretics). His Child Pugh score was 13 (Class C) and MELD was 39. The marker of acute phase reaction such as C-Reactive protein (CRP) was not available.

His chest X-ray showed left-sided pleural effusion. A CT thorax revealed bilateral patchy ground glass attenuation of the lung parenchyma (Fig. 1a). His nasopharyngeal swab for SARS-CoV-2 RT-PCR was positive. Ascitic fluid analysis did not show spontaneous bacterial peritonitis. He was commenced on hydroxychloroquine and azithromycin which was the then protocol for management of COVID-19 patients. However, his clinical condition deteriorated with hemodynamic instability and worsening hypoxia requiring ventilatory support. Patient died on day 4 following the diagnosis.

Case 2 A 47-year-old gentleman was examined in December 2019. He had decompensated cryptogenic cirrhosis with serum bilirubin of $12.2 \mathrm{mg} / \mathrm{dl}$, ALT $70 \mathrm{U} / \mathrm{L}$, INR 1.82 , albu$\min 2.5 \mathrm{~g} / \mathrm{dl}$ and creatinine $0.58 \mathrm{mg} / \mathrm{dl}$. He was negative for Hepatitis B including anti-HBcAb and anti-HCV antibodies. His non-invasive liver screen did not show metabolic or autoimmune etiology. There was no significant past medical history. He was advised work up for liver transplantation, but there was some delay in the process due to COVID-19 pandemic. He re-presented at the end of March 2020 with jaundice and abdominal distension. He was breathless with a respiratory rate 32 per minute. He was jaundiced, had tense ascites with no encephalopathy. His oxygen saturation was $89 \%$ on room air. His blood tests showed $\mathrm{Hb} 12.1 \mathrm{~g} / \mathrm{dl}$, white cell count 6530 cells $/ \mathrm{mm}^{3}$ with neutrophils 5550 cells $/ \mathrm{mm}^{3}$ (85\%) and lymphocytes 457 cells $/ \mathrm{mm}^{3}$ (7.0\%) and platelet count $114 \times 10^{9} / \mathrm{L}$. His serology was negative for Hepatitis $\mathrm{A}$ and $\mathrm{E}$ IgM. His LFT showed a significant deterioration with bilirubin $21.6 \mathrm{mg} / \mathrm{dl}$, ALT 70 U/L, INR 3.36, creatinine $0.97 \mathrm{mg} / \mathrm{dl}$, with MELD of 32. His CRP was $12.4 \mathrm{mg} / \mathrm{L}$. 


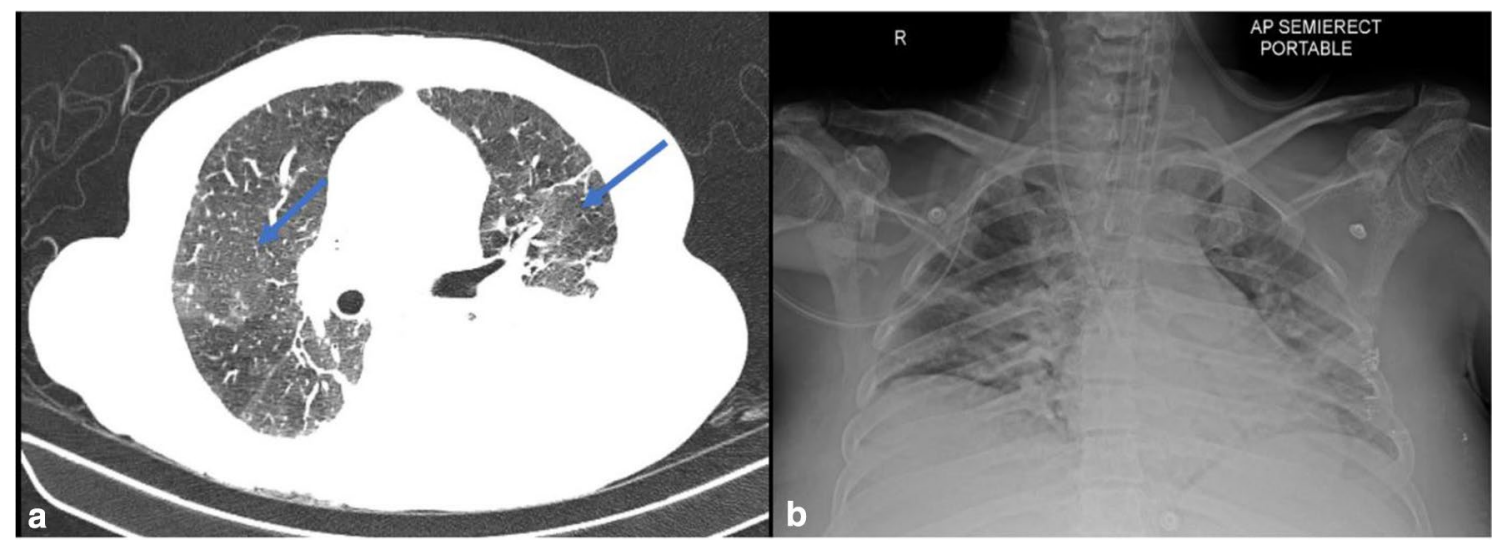

Fig. 1 a (Case 1): CT thorax showing bilateral patchy ground glass attenuation of the lung parenchyma with interstitial edema and left sided pleural effusion. b (Case 2): Chest X-ray showing features of bilateral pulmonary infiltrates

His chest X-ray showed mild bilateral patchy opacities. In view of tense ascites and respiratory distress, a large volume paracentesis was performed but with no improvement in his symptoms. His CT thorax showed diffuse areas of mosaic attenuation changes in both lungs. His nasopharyngeal swab for SARS-CoV-2 RT-PCR was initially negative. However, his condition continued to deteriorate with hepatic encephalopathy and hypotension requiring admission to intensive care. His repeat chest X-ray showed worsening bilateral pulmonary infiltrates (Fig. 1b). A repeat RT-PCR was positive for SARS-CoV-2. Unfortunately, his liver and hemodynamic parameters deteriorated with increasing oxygen requirement needing invasive ventilation. He died of multiorgan failure 3 days after the 2 nd COVID-19 test.

\section{Discussion}

These two cases possibly illustrate the adverse effect of SARS-CoV-2 in patients with decompensated cirrhosis.

Both these patients developed significant liver injury probably related to SARS-CoV-2, leading to jaundice, coagulopathy, ascites and hepatic encephalopathy, fulfilling the APASL definition for ACLF. Subsequently, their condition worsened with hemodynamic instability and progressive hypoxia leading to untimely death. Liver is involved in COVID-19 probably by direct cytopathic effect or possibly by cytokine mediated injury. Interestingly, ACE2 the receptor that binds SARS-CoV-2 is highly expressed in cholangiocytes $(59.7 \%)$ and in hepatic vasculatures. Elevated liver enzymes in COVID-19 were observed more in critically ill patients. Hepatic dysfunction in these patients was associated with poor prognosis [3]. Clinical deterioration with worsening of jaundice, ascites leading to ACLF in our cases were probably linked to COVID-19. There was no other attributable precipitating cause that we could identify.
A transjugular liver biopsy may have been useful, unfortunately, both patients were clinically unstable to undergo the procedure.

Wang and colleagues [4] described findings from postmortem liver biopsy in two patients with severe COVID-19. The liver histology showed substantial apoptosis of hepatocytes with mild-to-moderate focal lobular inflammation and lymphocytic infiltration. More importantly, electron microscopy identified large number of coronavirus particles within the cytoplasm of hepatocytes with associated mitochondrial edema and damaged cell membrane. Treatment of COVID19 continues to evolve. Based on recent trials HCQ is no longer recommended.

Azithromycin, a macrolide antibiotic used in COVID-19 to prevent bacterial infections, is metabolized in the liver and, therefore, should be used with caution in cirrhotic patients.

Both our patients developed worsening hemodynamics and altered neurological status, the cause of which could be multitude. Most likely reason in this scenario is hepatic encephalopathy related to worsening liver failure secondary to COVID-19. However, SARS-CoV-2 related neurotoxicity could not be ruled out. A recent systematic review reported at least 25\% COVID-19 patients develop neurological complication, manifesting as headache and altered sensorium [5]. Alternatively, hypoxia and multiorgan failure related to liver failure could also mimic hepatic encephalopathy. It is known that COVID-19 patients with underlying comorbid conditions are associated with poor survival. Patients with decompensated cirrhosis, in addition to underlying liver dysfunction, coexisting complications such as hypoalbuminemia, sarcopenia and frailty may influence the clinical outcome, particularly in the presence of superadded infections. Moreover, cirrhosis is characterised by increased systemic inflammation and immune paralysis at the same time, leading to complex immunological dysfunction. These factors 
may contribute to severe disease with rapid progression in patients with decompensated cirrhosis. Thus the presence of underlying cirrhosis is probably associated with much higher mortality in COVID-19 compared to other comorbidities.

To our knowledge, ours is the first reported cases of COVID-19 associated with ACLF fulfilling APASL criteria leading to rapid deterioration and death. A preliminary data from an international liver registry reported 152 cases of COVID-19 in patients with chronic liver disease with a higher morality in Child Pugh $\mathrm{C}$ disease. However, the median bilirubin in this series was $1.1 \mathrm{mg} / \mathrm{dl}$ with a MELD score of 10 and notably there was no reported ACLF [6]. Given the ongoing pandemic in several countries, it is important to obtain cases as much as possible for better management of these high--risk patients.

Author contributions MR (conceptualization, supervision, original write up and review). VP (writing- review and editing). GN (writing- review and editing). DJ (data curation, software, writing- review and editing).

\section{References}

1. Guan WJ, Ni ZY, Hu Y, et al. Clinical characteristics of coronavirus disease 2019 in China. N Engl J Med
2020;382(18):1708-1720. https://doi.org/10.1056/NEJMoa2002 032

2. Grasselli G, Zangrillo A, Zanella A, et al. Baseline Characteristics and Outcomes of 1591 Patients Infected With SARS-CoV-2 Admitted to ICUs of the Lombardy Region, Italy [published online ahead of print, 2020 Apr 6]. JAMA 2020;323(16):15741581. https://doi.org/10.1001/jama.2020.5394

3. Jothimani D, Venugopal R, Abedin MF, Kaliamoorthy I, Rela M. COVID-19 and Liver [published online ahead of print, 2020 Jun 15]. J Hepatol 2020. https://doi.org/10.1016/j.jhep.2020.06.006

4. Wang Y, Liu S, Liu H, et al. SARS-CoV-2 infection of the liver directly contributes to hepatic impairment in patients with COVID-19 [published online ahead of print, 2020 May 10]. J Hepatol 2020. https://doi.org/10.1016/j.jhep.2020.05.002

5. Asadi-Pooya AA, Simani L. Central nervous system manifestations of COVID-19: a systematic review. J Neurol Sci 2020;413:116832. https://doi.org/10.1016/j.jns.2020.116832

6. Moon A, Webb G, et al. High mortality rates for SARS-CoV-2 infection in patients with pre-existing chronic liver disease and cirrhosis: preliminary results from an international registry. J Hepatol 2020. https://doi.org/10.1016/j.jhep.2020.05.013

Publisher's Note Springer Nature remains neutral with regard to jurisdictional claims in published maps and institutional affiliations. 